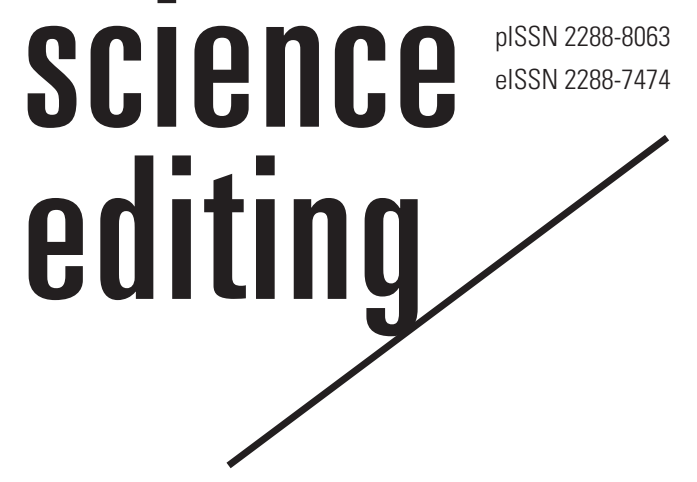

\title{
Corrigendum
}

\section{Restriction of some datasets from open access repositories}

\author{
Editorial Office, Korean Council of Science Editors
}

It has been informed that Clarivate Analytics does not allow the data downloaded from the Web of Science Core Collection or their derivatives to be posted on open access data repositories. Therefore, the approach to the dataset files of the following articles in the Havard Dataverse has been restricted:

Hsin-Luen Tsai, Jia-Fen Wu. Bibliometric analysis of flipped classroom publications from the Web of Science Core Collection published from 2000 to 2019. Sci Ed. 2020;7(2):163-168. https://doi.org/10.6087/kcse.212

Juyeon Oh, Aekyung Kim. A bibliometric analysis of COVID-19 research published in nursing journals. Sci Ed. 2020;7(2):118-124. https://doi.org/10.6087/kcse.205 